\title{
Knowledge management for multi- and crossdisciplinary research projects
}

\author{
Pieter J. Beers \\ Delft University of Technology \\ p.j.beers@tudelft.nl
}

\author{
Pieter W. G. Bots \\ Cemagref \\ pieter.bots@cemagref.fr
}

Theories on knowledge management (KM) have matured to include organizational practices, information systems and knowledge processes. Sharing of knowledge between actors having different (scientific) perspectives has received relatively little attention, whereas this is especially relevant to large-scale multidisciplinary research projects. In such projects, the actors are academics, the knowledge content is diverse and shaped (and often confined) by scientific disciplines, whereas strategies to cross disciplinary boundaries seem to be lacking. These projects face the challenge of overcoming disciplinary and theoretical differences in order to reap the benefits of crossdisciplinary inquiry. This minitrack focuses on the management of knowledge within large-scale multidisciplinary and crossdisciplinary research projects.

How can we compare results obtained using theories and models from different disciplinary backgrounds? Work done within two different modeling approaches may still be well comparable, even though the difference in research approach might suggest otherwise. Likewise, research done within the context of one approach may still make use of different, incomparable (and possibly even incompatible) techniques, even though the similarity in approaches might suggest otherwise. We are therefore in search of ways to detect fundamental (i.e., irreducible) differences between disciplinary theories and models, ways to avert the risk of reinventing the wheel, and ways to prevent scientific oversight of issues that should receive scientific scrutiny.

How to position this minitrack in the field of knowledge management research? This by now vast field - its growth is mirrored by the evolution of the HICSS KM track - can be seen to address both content and process. Content here refers to the creation of shared semantics using ontologies as well as methods and tools to codify, store, and disseminate knowledge. Process refers to the cognitive processes of developing shared meaning as well as the organizational processes needed to achieve (more) effective creation and use of knowledge. We expect that new insights, models, methods, and tools that are developed for content and process throughout the $\mathrm{KM}$ field can find useful application in the organization and management of scientific research projects.

The content of this minitrack has turned out somewhat broader, in terms of field of application, than originally intended. Apparently, interdisciplinary research projects as a field of interest currently receive little attention from the KM community. Nonetheless, the minitrack features three interesting papers.

Khalifa, Yan Yu and Ning Shen border on our topic when one regards scientific discovery as innovation, and a research team as an organization. They present an integrated view on organizational innovativeness. Their research shows that usage of knowledge management systems, organizational learning processes, and proactive strategic orientation are important influences on organizational innovativeness. The question whether these findings can be translated to the context of interdisciplinary research projects provides an interesting topic for discussion.

Zimmer and Henry found that the use of an information source depends not only on accessibility and quality of that source, but also on job and individual characteristics of the information searcher. Here, we would like to discuss the characteristics of scientists, and the implications of the presenters' findings for the organization of interdisciplinary projects.

We ourselves propose conceptual analysis as a method to identify, and subsequently deal with, differences in conceptual models constructed by researchers with different disciplinary backgrounds who work together in interdisciplinary research projects. We look forward to a discussion with $\mathrm{KM}$ experts, in particular on the efficiency of the method we propose.

As KM itself is an interdisciplinary field of research, the ideas put forward by the presenters should apply to our own knowledge work We therefore trust that the discussions will be lively. 